\title{
Buoyancy induced heat transfer deterioration in vertical concentric and eccentric annuli
}

\author{
Pourya Forooghi ${ }^{a}$, Iman Ashtiani Abdi ${ }^{a}$, Mahidzal Dahari ${ }^{\mathrm{b}}$, Kamel Hooman ${ }^{\mathrm{a}, *}$ \\ a Queensland Geothermal Energy Centre of Excellence, School of Mechanical and Mining Engineering, University of Queensland, QLD 4072, Australia \\ bepartment of Mechanical Engineering, Faculty of Engineering, University of Malaya, 50603 Kuala Lumpur, Malaysia
}

A R T I C L E I N F O

\section{Article history:}

Received 1 May 2014

Received in revised form 6 September 2014

Accepted 3 October 2014

Available online 4 November 2014

\section{Keywords:}

Heat transfer deterioration

Heat transfer deterioration
Turbulent mixed convection

Eccentric annuli

\begin{abstract}
A B S T R A C T
Turbulent convection heat transfer of upward fluid flows in vertical annular channels with uniformly heated inner wall and adiabatic outer wall is investigated numerically. Two concentric geometries with outer-to-inner diameter ratios of 2.4 and 3 as well as two eccentric geometries with identical outer-toinner diameter ratio of 2.4 and eccentricities of 0.25 and 0.5 are investigated. Heat transfer deterioration, similar to that of a circular pipe, is observed. The range of buoyancy parameter, over which deterioration is observed, is close to that of circular pipes. Moreover, it is observed that when the value of eccentricity increases, the deterioration phenomenon becomes less pronounced and recovery of heat transfer coefficient occurs earlier, i.e. at lower buoyancy parameters compared to concentric annuli.
\end{abstract}

(c) 2014 Elsevier Ltd. All rights reserved.

\section{Introduction}

Variation of the thermophysical properties can significantly affect transport phenomena in fluid flows. A good example is the case of fluids at pressures slightly higher than critical pressure when rapid variations in the values of specific heat, density, viscosity and thermal conductivity against temperature is present [1]. In such fluid flows, if the fluid exchanges heat with a solid wall, property variations can be significant in the normal-to-wall direction. If one intends to correctly account for heat transfer coefficient in such flows, correction factors are required for the wall-to-bulk values of thermal properties [2,3].

Variation of density can affect heat transfer also in a rather indirect manner, which is, causing significant buoyancy forces thereby changing the fluid velocity field. A notable phenomenon stemming from this boost of buoyancy force is what commonly referred to as "heat transfer deterioration" or briefly "deterioration". Deterioration, which is simply a sudden reduction in the value of heat transfer coefficient, is likely to occur whenever the regime is turbulent, the flow direction is vertical and in the same direction as the buoyancy force adjacent to the wall, i.e. upward heating or downward cooling. In 1979, Jackson and Hall [4] explained this phenomenon as the indirect impact of buoyancy on the production of turbulence kinetic energy by deforming the velocity profile in a channel. According to their explanation, in an upward flow, heated

\footnotetext{
* Corresponding author. Tel.: +61 7 33653677; fax. +61 733654799.

E-mail address: k.hooman@uq.edu.au (K. Hooman).
}

fluid near the wall tends to move upward faster because it is lighter. As a result, the velocity profile differs from that of no-buoyancy flow so that the velocity gradient decreases in the near-wall region where turbulence production would have been significant. It reduces the level of turbulence intensity and, consequently, heat transfer is deteriorated. This theory was later supported by flow structure observations of Kurganov and Kaptilnyi [5]. Aiming to predict the onset of deterioration, Jackson et al. [6] introduced a buoyancy parameter $(B o)$ to quantify the buoyancy effect:

$B o=\frac{G r}{R e^{3.425} \operatorname{Pr}^{0.8}}$

where

$G r=\frac{\rho_{b}^{2} g \beta_{b} q_{w} D_{h}^{4}}{\mu_{b}^{2} \lambda_{b}}$

Reynolds number should be similarly defined based on hydraulic diameter $D_{h}$. For Reynolds number, the properties are calculated in bulk temperature. When the above buoyancy parameter reaches a certain threshold, sudden decrease in the value of heat transfer coefficient, or Nusselt number, is observed. It must be noted that with very high values of buoyancy parameter, heat transfer starts to recover as a result of free convection becoming dominant. This 'deterioration' and 'recovery' sequence can be seen in Figure, which will be further discussed in Section 3 .

In order to better study deterioration phenomena and its dependence on the above mentioned buoyancy parameter or similar ones, a number of experimental studies have been conducted. 


\begin{tabular}{|c|c|c|c|}
\hline \multicolumn{4}{|c|}{ Nomenclature } \\
\hline \multicolumn{2}{|c|}{ Latin symbols } & $x, y, z$ & Coordinates (m) \\
\hline Bo & buoyancy parameter & & \\
\hline$C_{p} \quad s$ & specific heat at constant pressure $\left(\mathrm{W} \mathrm{kg}^{-1} \mathrm{~K}^{-1}\right)$ & \multicolumn{2}{|c|}{ Greek symbols } \\
\hline \multirow{2}{*}{$\begin{array}{l}C_{\mu, \varepsilon, 1,2, \eta, L} \\
D\end{array}$} & model constant & $\beta$ & volumetric expansion coefficient $\left(\mathrm{K}^{-1}\right)$ \\
\hline & diameter (m) & $\epsilon$ & dissipation rate of turbulence energy $\left(\mathrm{m}^{2} \mathrm{~s}^{-3}\right)$ \\
\hline \multirow{3}{*}{$\begin{array}{l}e \\
f \\
G_{k}\end{array}$} & eccentricity & $\varphi$ & non-dimensional temperature \\
\hline & production of turbulence velocity scale $\left(\mathrm{s}^{-1}\right)$ & $\theta$ & angle with $y$ axis in $x-y$ plane (rad) \\
\hline & production of turbulence energy due to density fluctua- & $\lambda$ & thermal conductivity $\left(\mathrm{W} \mathrm{m}^{-1} \mathrm{~K}^{-1}\right)$ \\
\hline & tion $\left(\mathrm{m}^{2} \mathrm{~s}^{-3}\right)$ & $\mu$ & kinetic viscosity (Pa s) \\
\hline$\vec{g}$ & gravitational acceleration vector $\left(\mathrm{m} \mathrm{s}^{-2}\right)$ & $v$ & kinematic viscosity $\left(\mathrm{m}^{2} \mathrm{~s}^{-1}\right)$ \\
\hline Gr & Grashoff number & $\rho$ & density $\left(\mathrm{kg} \mathrm{m}^{-3}\right)$ \\
\hline$h$ & enthalpy $\left(\mathrm{J} \mathrm{kg}^{-1}\right)$ & $\sigma$ & turbulent Prandtl number \\
\hline$k$ & turbulence kinetic energy $\left(\mathrm{m}^{2} \mathrm{~s}^{-2}\right)$ & & \\
\hline$L$ & length scale $(\mathrm{m})$; see Eq. (12) & Subscri & \\
\hline$l$ & distance between tube centers (m) & ave & area-averaged on a periphery \\
\hline $\mathrm{Nu}$ & Nusselt number & $b$ & bulk value \\
\hline$P_{k}$ & production of turbulence energy due to shear $\left(\mathrm{m}^{2} \mathrm{~s}^{-3}\right)$ & $F C$ & forced convection \\
\hline$p$ & pressure $(\mathrm{Pa})$ & $h$ & hydraulic \\
\hline $\operatorname{Pr}$ & Prandtl number & $i$ & inner \\
\hline & heat flux $\left(\mathrm{W} \mathrm{m}^{-2}\right)$ & mean & mass-averaged over a cross section \\
\hline $\operatorname{Re}$ & Reynolds number & 0 & outer \\
\hline$r$ & radial coordinate measured from pipe centreline (m) & ref & reference value \\
\hline$S$ & strain tensor & & turbulence \\
\hline$T$ & temperature $(\mathrm{K})$ & & measured on the wall \\
\hline & time scale (s); see Eq. (6) & z & axial component \\
\hline$\vec{U}$ & velocity vector & & \\
\hline$U$ & velocity magnitude $\left(\mathrm{m} \mathrm{s}^{-1}\right)$ & & \\
\hline $\bar{v}^{2}$ & turbulence velocity scale $\left(\mathrm{m}^{2}\right)$ & & \\
\hline
\end{tabular}

Watts and Chou [7] derived a correlation to predict deterioration based on the buoyancy parameter for supercritical water in circular pipes. Similar studies have been done for circular pipes with $\mathrm{CO}_{2}[1,8]$.

Attempts have also been made to simulate deterioration phenomenon numerically. As this phenomenon is closely related to the mechanism by which turbulence energy is produced, the right choice of turbulence model is crucial here. A widespread choice for this problem has been low Reynolds variations of $k-\varepsilon$ model [9-11]. However more careful studies have recently revealed that this class of two-equation models may not be as loyal to the physics of problem as their results suggest. The same references recommend a more elaborated four equation model of $k-\varepsilon-v^{2}-f[12]$ to be the best choice within the category of eddy viscosity models [10,13-15].

A big difficulty with the study of deterioration is that deterioration is only one of the phenomena arisen as a result of rapid property variations so it is not easy to distinguish the pure effect of buoyancy on heat transfer when real fluids are studied. To tackle this problem, a number of researchers adopted constant property assumption along with Boussinesq approximation for the calculation of buoyancy term in the momentum equation $[13,16]$. This computational approach facilitates study of the pure effect of buoyancy on transport phenomena in turbulent flows.

A careful literature review reveals that most of the references (numerical or experimental) have studied the deterioration phenomenon using a circular pipe in vertical direction as the geometry, with exception of [17], studying it an inclined circular pipe. Few researches have been done on the heat transfer of supercritical fluids in 'concentric' annular pipes [18-20], none of them has studied the effect of buoyancy - isolated from other effects. On 'eccentric' annular pipes, however, no research has been reported so far.
The present paper, aims to investigate the deterioration phenomenon in both concentric and eccentric annuli using Boussinesq assumption in order to isolate the effect of buoyancy, as described above. The focus of this study will be on the effect of buoyancy parameter on heat transfer and the trend of deterioration phenomenon with the eccentricity of the annulus.

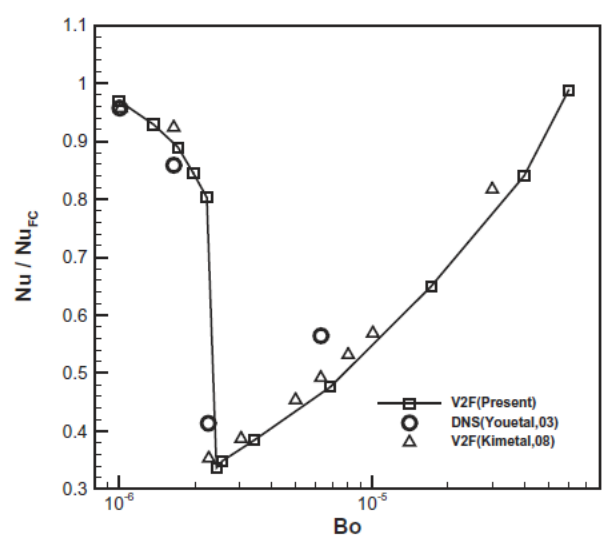

Fig. 1. Comparison of the present solution with DNS data of [16] and a separate CFD simulation [13] using the same turbulence model. The Nusselt number is for upward flow in a circular pipe with $R e=5300$. Boussinesq assumption has foen adopted in all three studies. The deterioration occurs at $B O=2 \times 10^{-6}$ followed by a recovery zone (monotonic increase in $\mathrm{Nu} / \mathrm{Nu} u_{F C}$ ). 
Table 1

Model constants.

\begin{tabular}{llllllllll}
\hline$C_{\mu}$ & $C_{1 \epsilon}$ & $C_{2 \epsilon}$ & $C_{1}$ & $C_{2}$ & $C_{1 \eta}$ & $C_{L}$ & $\operatorname{Pr}_{t}$ & $\sigma_{k}$ & $\sigma_{\epsilon}$ \\
\hline 0.22 & 1.4() & & & & & & & &
\end{tabular}

\section{Mathematical modeling}

The problem under consideration is schematically depicted in Fig. 1. The solution domain is the space between the inner and outer cylinders. Governing equations for momentum, turbulence and energy transport were solved in the three-dimensional domain; length of the channel in axial direction is $0.3 \mathrm{~m}$. Uniform velocity and temperature profiles were used, so the flow is developing inside the pipe for a certain distance from the inlet, as will be discussed in results and discussion.

Continuity:

$\nabla \cdot \vec{U}=0$

Momentum:

$\nabla \cdot(\vec{U} \vec{U})=-\frac{\nabla P}{\rho}+\vec{g} \beta\left(T-T_{\text {ref }}\right)+\nabla \cdot\left(2\left(v+v_{t}\right) S\right)$

where $\vec{U}$ is the Reynolds averaged velocity vector and $S=\frac{1}{2}$ $\left(\nabla \vec{U}+\nabla \vec{U}^{T}\right)$

Energy:

$$
\nabla \cdot\left(\vec{U} C_{P} T\right)=\nabla \cdot\left(\left(\frac{v}{P r}+\frac{v_{t}}{P r_{t}}\right) \nabla T\right)
$$

$v_{t}$ is turbulence kinetic viscosity and is defined as [12]:

$v_{t}=C_{\mu} \bar{v}^{2} \tilde{t}, \quad \tilde{t}=\max \left(\frac{k}{\epsilon}, 6 \sqrt{\frac{v}{\epsilon}}\right)$,

which can be calculated after solving the following turbulence transport equations:

Turbulence kinetic energy:

$\nabla \cdot(\vec{U} k)=\nabla \cdot\left(\left(v+\frac{v_{t}}{\sigma_{k}}\right) \nabla k\right)+P_{k}+G_{k}-\epsilon$

Turbulence dissipation:

$\nabla \cdot(\vec{U} \epsilon)=\nabla \cdot\left(\left(v+\frac{v_{t}}{\sigma_{\epsilon}}\right) \nabla \epsilon\right)+C_{\epsilon 1} \frac{P_{k}+G_{k}}{\tilde{t}}-C_{\epsilon 2} \frac{\epsilon}{\tilde{t}}$

Turbulence velocity scale $\left(\bar{v}^{2}\right)$ :

$\nabla \cdot\left(\vec{U} \bar{v}^{2}\right)=\nabla \cdot\left(\left(v+\frac{v_{t}}{\sigma_{k}}\right) \nabla \bar{v}^{2}\right)+k f-\bar{v}^{2} \frac{\epsilon}{k}$

Relaxation equation:

$$
f-L^{2} \nabla^{2} f=\left(C_{1}-1\right) \frac{\left(2 / 3-\bar{v}^{2} / k\right)}{\bar{t}}+C_{2} \frac{\left(P_{k}+G_{k}\right)}{k}
$$

In the above equations $P_{k}$ and $G_{k}$ stand for production of $k$ due to shear and buoyancy, respectively, and $L$ is the turbulence length scale:

$P_{k}=2 v_{t}\left(S^{2}\right)$

$G_{k}=\beta \frac{v_{t}}{\operatorname{Pr}}(\vec{g} . \nabla T)$

$L=C_{L} \max \left(\frac{k^{3 / 2}}{\epsilon}, C_{\eta}\left(\frac{\nu^{3}}{\epsilon}\right)^{1 / 4}\right)$

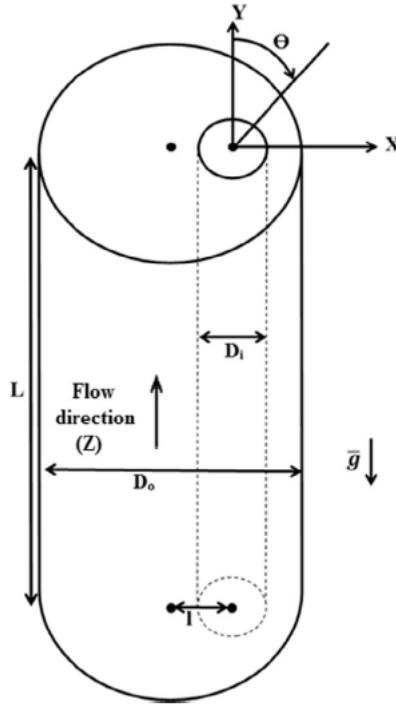

Fig. 2. Schematic of an annulus cross section ( $X$ and $Y$ are in the cross section plane).
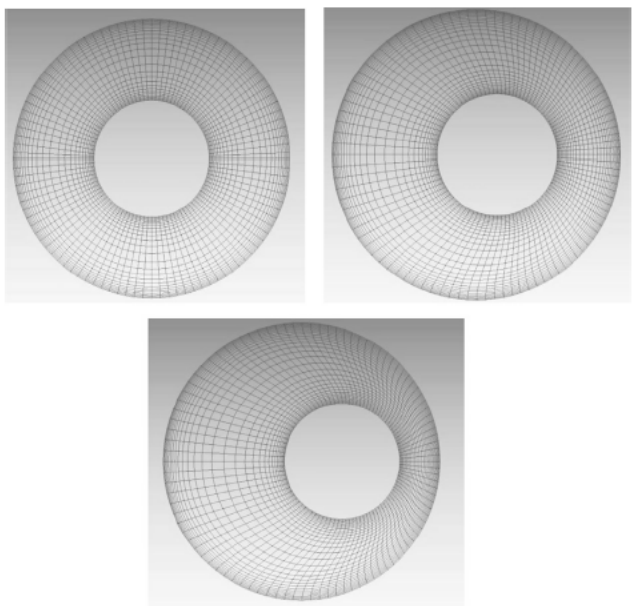

Fig. 3. Snapshots of the computational grids ( $X-Y$ plane).

As already mentioned, in this paper, constant properties are assumed along with Boussinesq approximation for Archimedes' force term in the momentum equation in order to isolate the effects of buoyancy from other effects arising due to property variation. The following constant values are used for the properties. These values are chosen close to those of $\mathrm{CO}_{2}$ around its pseudo-critical point in $8.0 \mathrm{MPa}$, i.e. slightly above its critical pressure (7.4 MPa):

$\rho=300\left(\mathrm{~kg} \mathrm{~m}^{-3}\right) \lambda=0.06\left(\mathrm{~W} \mathrm{~m}^{-1} \mathrm{~K}^{-1}\right) \mu=5 \times 10^{-5}$ (Pa s) $\beta$

$=0.05\left(K^{-1}\right)$

Pseudo-critical point is a point where the variation of density is most rapid; hence deterioration is most likely to happen. 

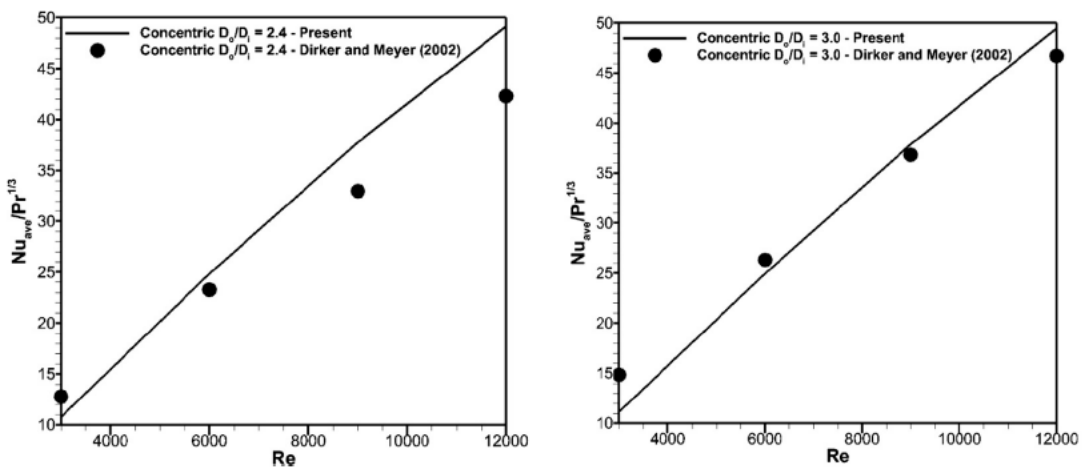

Fig. 4. Validation of the present results against an empirical correlation for concentric annuli with diameter ratios of 2.4 (left) and 3.0 (right).
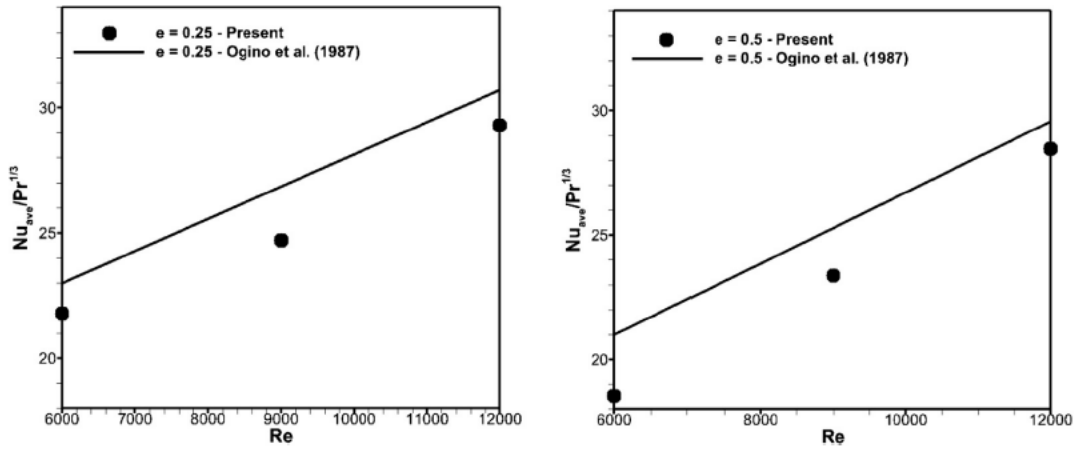

Fig. 5. Validation of the present results against an experiment for eccentric annuli with eccentricity ratios of 0.25 (left) and 0.5 (right). The same boundary condition as that used in the reference paper, i.e. constant temperature on inner and outer walls, have been applied in the present simulation for the purpose of validation.
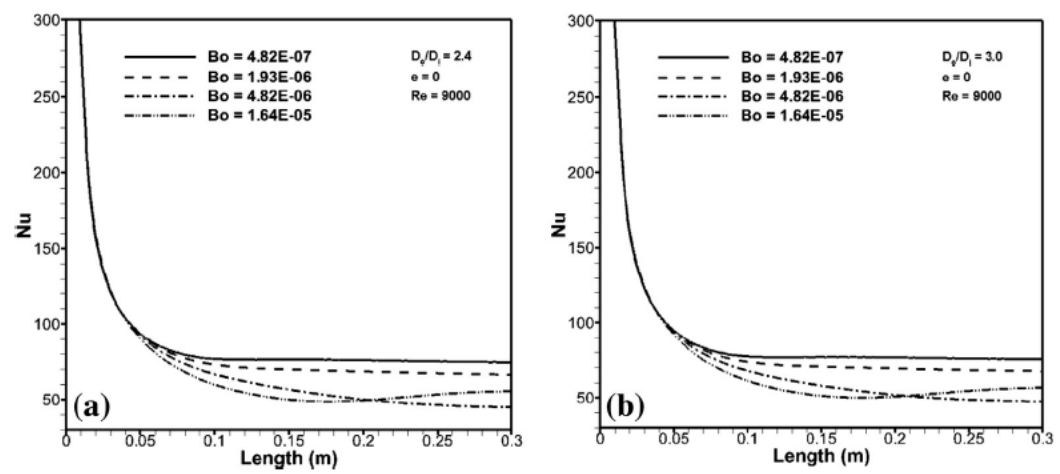

Fig. 6. Variation of Nusselt number in axial direction for concentric cases. Reynolds number is equal to 9000 and the diameter ratio is equal to 2.4 (a) and 3 (b).

Boundary conditions: All transport equations are solved all way down to the solid wall so there is no need to use wall functions. No-slip condition is applied on the solid walls, i.e. all velocity components are zero on the walls. For temperature, constant heat flux and adiabatic boundary conditions are used for inner and outer walls, respectively. Turbulence kinetic energy and velocity scale $\left(\bar{v}^{2}\right.$ are both zero on the walls. Furthermore, dissipation rate must satisfy the following equation on the wall: $\epsilon_{w}=\left.\frac{\partial^{2} k}{\partial n^{2}}\right|_{w}$

For the inlet boundary, uniform values are used for all quantities; for velocity, non-axial components are zero on the inlet. Inlet temperature is set at $25^{\circ} \mathrm{C}$. For the outlet boundary, derivatives of all variables with respect to the axial direction are set to zero.

The values of model constants are presented in Table 1. 

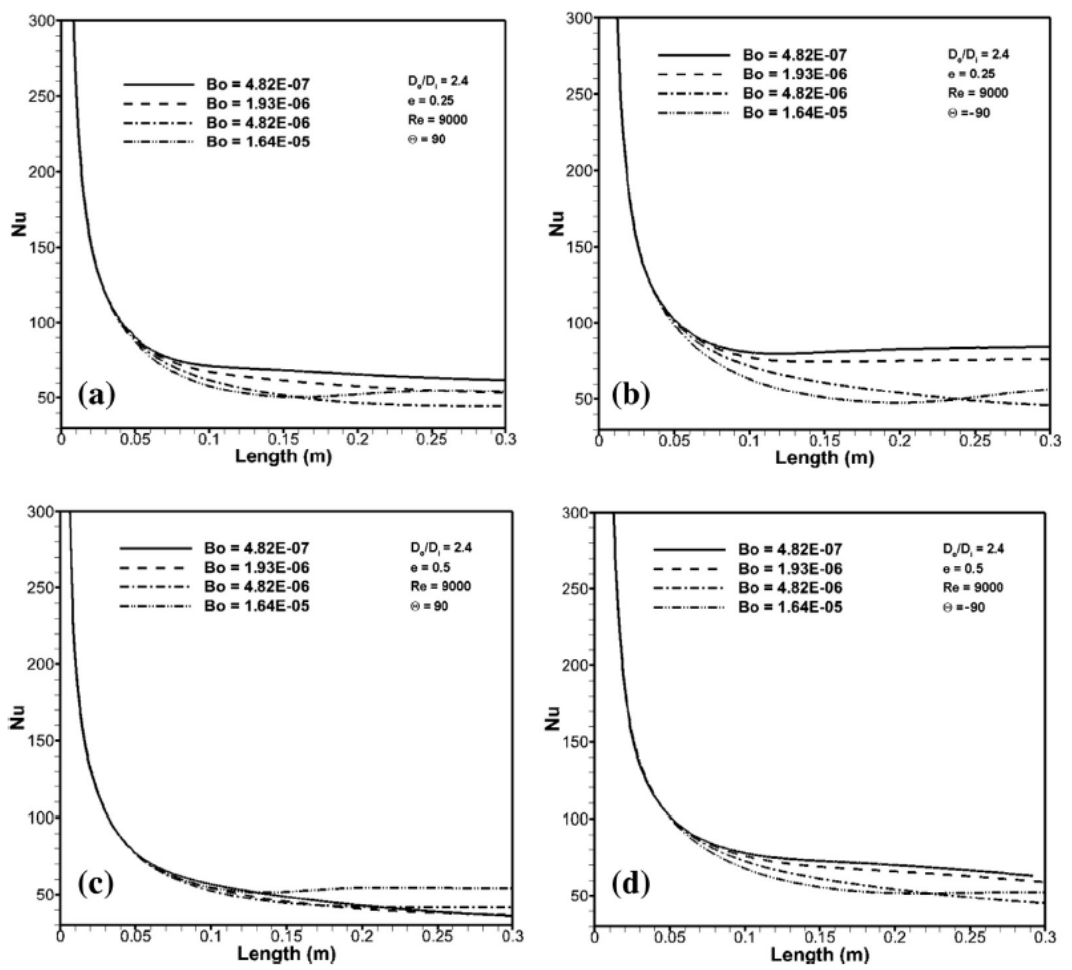

Fig. 7. Variation of Nusselt number in axial direction for eccentric cases. Reynolds number is equal to 9000 . (a) and (b) shows the Nusselt number for the annuli with the eccentricity equal to 0.25 in $\theta$ equal to 90 and -90 , respectively. (c) and (d) contains similar data for the annuli with the eccentricity equal to 0.5 .

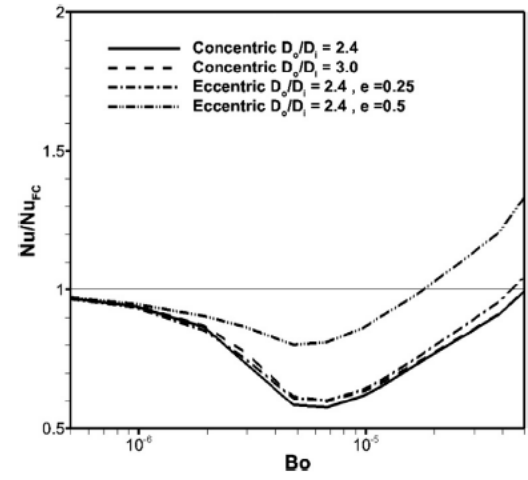

Fig. 8. Variation of mixed-to-forced convection Nusselt number against Buoyancy parameter $(B o)$ for four different annuli; $R e=9000$

\section{Solution of governing equations}

\subsection{Numerical scheme}

General purpose commercial CFD code FLUENT was used to solve the governing equations. SIMPLE algorithm was employed in order to couple continuity and momentum equations and second order upwind scheme was used for spatial discretization A mapped numerical grid with dimensions of $30 \times 100 \times 1000$ in radial, tangential and axial direction is adopted. Near both solid walls boundary layer grids are used with the first grid point thickness of $0.03 \mathrm{~mm}$. The mesh independence of the solution was examined comparing with a finer computational grid with dimensions of $50 \times 150 \times 1500$, and the difference in the computed values of heat transfer coefficient was less than $0.2 \%$. Fig. 2 depicts the computational grids used in this work. The values of first grid point $\mathrm{y}^{+}$in the chosen mesh are between 1 and 2.5; larger values up to 6 rarely occur in positions with lower wall shear stress.

\subsection{Validation of results}

The numerical solution has been benchmarked for its capability of capturing 'deterioration' due to buoyancy. To do that, DNS data of You et al. [16] are used. This reference has used Boussinesq assumption along with constant properties that makes it suitable for the validation of the present data.

In this paper Nusselt number is defined as:

$$
N u=\frac{q \cdot D_{h}}{\lambda_{b}\left(T_{w}-T_{b}\right)}
$$

Figure shows the variation of $N u / N u_{F C}$ against $B o . N u$ is the Nusselt number calculated for each particular value of $B o$ and $N u_{F C}$ is the forced convection Nusselt number that is calculated when the buoyancy term is set to zero. This type of diagram is widely used for assessing the effect of buoyancy on turbulence convection heat 

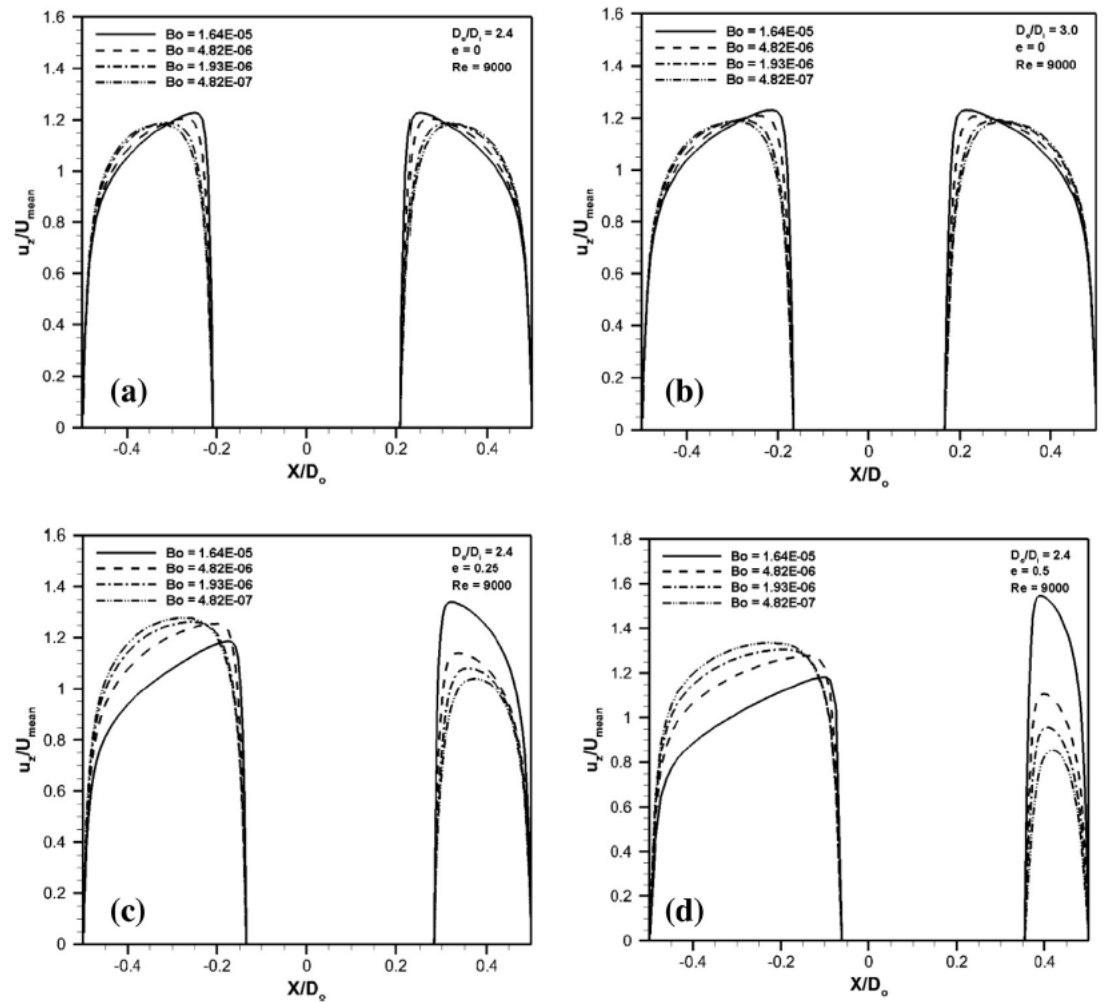

Fig. 9. Velocity profiles along $x$ direction for four different studied annuli. (a) Concentric with $D_{o} / D_{i}=2.4$; (b) concentric with $D_{o} / D_{i}=3.0$; (c) eccentric with $D_{o} / D_{i}=2.4$ and $e=0.25$; (d) eccentric with $D_{o} / D_{i}=2.4$ and $e=0.5$.

transfer $[1,7,8]$. It is observed that there is a good agreement between the current CFD solution and the DNS data, and both deterioration and recovery zones have been captured well in the present solution. It suggests that $k-\varepsilon-v^{2}-f$ model is doing well in predicting the buoyancy effects. It must be mentioned that the calculated values of $\mathrm{Nu}_{F C}$ were compared with the prediction of Petukhov-Gnielinski correlation [21] and were found to be within 5\% accuracy. More detailed benchmarking of the numerical model with regards to buoyancy effects has been reported in [17]. It must be mentioned that, in the present paper, the value of $B o$ is changed by changing heat flux in Eq. (2), while other parameters are kept constant.

The model is also validated for forced convection in concentric and eccentric annuli using the empirical correlation of Dirker and Meyer [22] and Ogino et al. [23], and the results are presented in Figs. 3 and 4. In Fig. 4, it is observed that the obtained values are some $10 \%$ below those suggested by the correlation. This is believed to be somehow due to the uncertainties in the experiments pointed out by [23]; in this reference it is mentioned that the flow might not be completely fully-developed in which case an overestimation of Nusselt number would be resulted. In addition, constant temperature boundary condition has been used for the wall in [23], which can be the origin of some minor discrepancies.

\section{Results and discussion}

The simulation has been done for four different annuli with identical hydraulic diameter of $5 \mathrm{~mm}$ and length of $0.3 \mathrm{~m}$ at
$R e=9000$. Two of the annuli are concentric with outer-to-inner diameter ratio $\left(D_{o} / D_{i}\right)=2.4$ and 3.0 . The other two are eccentric annuli with $D_{o} / D_{i}=2.4$ and eccentricity $(e)=0.25$ and 0.5 . Eccentricity is defined as:

$e=2 l /\left(D_{0}-D_{i}\right)$

where $l$ is the distance between the centers of inner and outer tubes as depicted in Fig. 1. Figs. 5 and 6 show the variation of the local Nusselt number on the inner wall of the annuli along the axial direction. The two graphs in Fig. 5 correspond to the concentric annuli and those in Fig. 6 correspond to the eccentric ones. Obviously, for the eccentric cases, Nusselt number is not uniform along the periphery. Here instead of averaged Nusselt numbers, local Nusselt numbers for two opposite locations $(\theta= \pm 90)$ on the wall, i.e. the widest and narrowest parts of the cross section are presented in order to better observe the trends. In each graph, four different values of $B o$ are examined.

Shortly downstream of the inlet, where hydrodynamic and thermal boundary layers are developing, the Nusselt numbers are very large for all cases. As forced convection boundary layers start to reach their ultimate thickness, Nusselt numbers start to put on constant values. However, in some of the curves, a slight increase is observed somewhere in the middle of the pipe. This secondary enhancement of Nusselt number, which is particularly significant in the curves corresponding to the highest Bo value, is a result of free convection becoming more significant and taking part in heat transfer. The fact that free convection boundary layer develops in a 

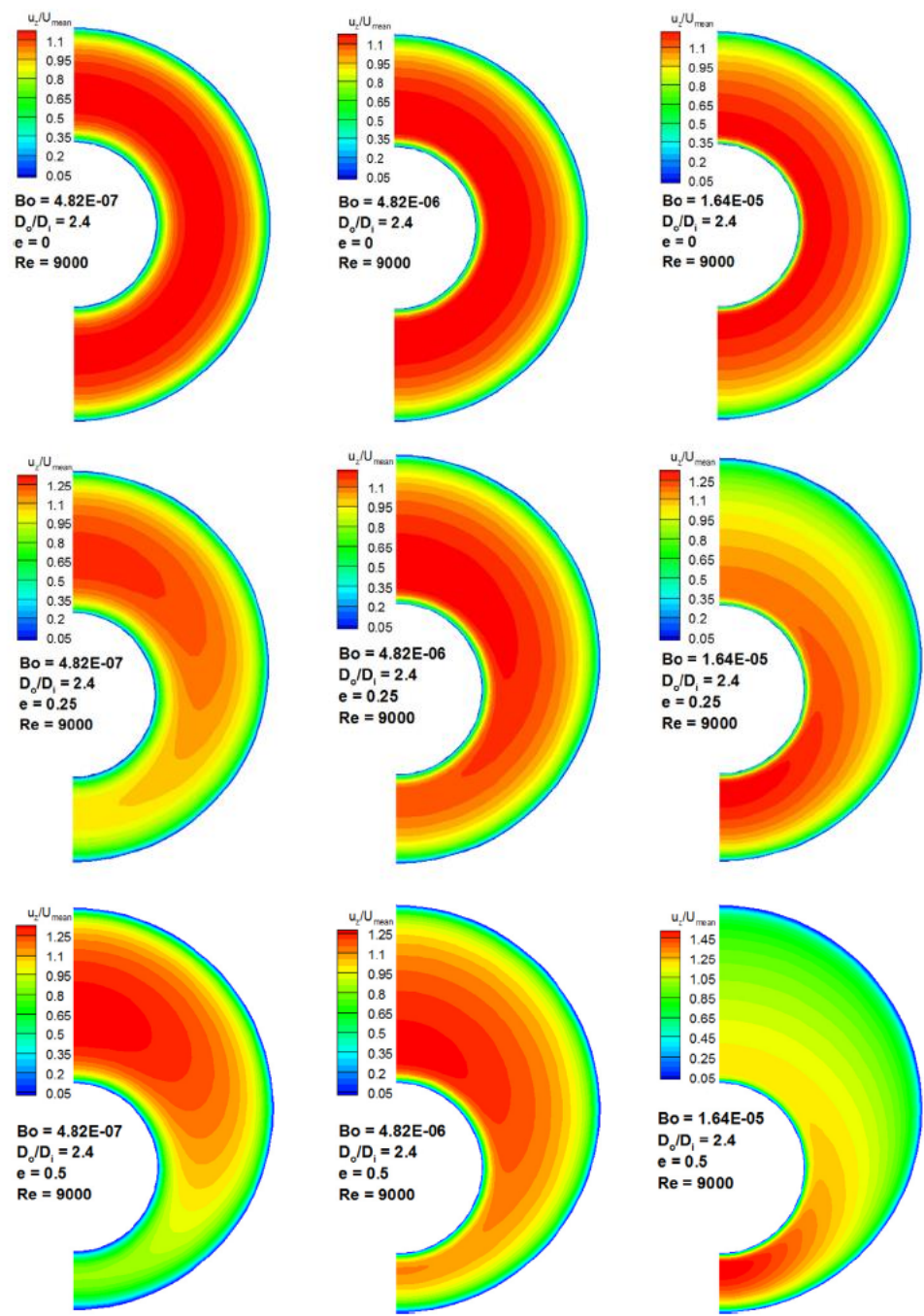

Fig. 10. Axial velocity distributions for annuli with $D_{o} / D_{i}=2.4$ and three different values of eccentricity. In each case, three different buoyancy conditions are considered: insignificant buoyancy (left), deterioration (middle) and recovery (right).

different rate from that of forced convection [24] explains why the increase in the Nusselt number due to free convection happen when forced convection boundary layer is almost fully developed. In all graphs - except Fig. 6(d), which corresponds to the largest studied value of eccentricity - the curve of the lowest Bo lies above other curves; as Bo increases, Nusselt number decreases all ove the pipe length except for the curve corresponding to the biggest Bo where Nusselt shows an increase due to free convection. The trend of Nusselt with Bo will be discussed in more details in the following paragraphs. Another point that is worth mentioning here is that the free convection boundary layer starts affecting heat transfer more upstream in $\theta=+90$ than in $\theta=-90$. It is obviously because the free convection boundary layer is first formed where the space between the walls is minimal.
In order to better investigate the effect of buoyancy, the ratio of $\mathrm{Nu} u_{\text {ave }}$ to $\mathrm{Nu} u_{F C \text {,ave }}$ has been calculated and depicted against buoyancy parameter for different geometries similar to what was done for a circular pipe in Figure. The data are obtained in the axial position of $0.3 \mathrm{~m}$ from the pipe inlet. Fig. 7 shows the summary of results for all four studied geometries. It is observed that, for all cases, a deterioration zone is formed in the same range of Bo where it has already been reported for circular pipes (compare with Figure). This deterioration zone is followed by a recovery zone, again, similar to circular pipes. The two concentric cases do not show any significant difference, and the eccentric case with the smaller value of eccentricity (0.25) also lies very close to them. However, the other eccentric annulus shows a considerably less severe deterioration and a quicker recovery.

Link to Full-Text Articles :

http://www.sciencedirect.com/science/article/pii/s0017931014008941 